\title{
El costo de la atención de las lesiones vasculares abdominales por proyectil de arma de fuego. Su impacto en el paciente, su familia y el hospital
}

\author{
The cost of gunshot abdominal vascular injuries. Its impact on patient, family and \\ hospital
}

\author{
Adriana Hernández-García*, Demian Trueba-Lozano y Juan C. Vázquez-Minero \\ Centro de Trauma, Cruz Roja Mexicana, Ciudad de México, México
}

\begin{abstract}
Resumen
Antecedentes: El trauma violento con lesiones penetrantes es un problema de salud pública relevante. Las heridas penetrantes abdominales causan el 90-95\% de las lesiones vasculares, las cuales tienen elevada morbilidad asociada y letalidad, e implican elevados costos de atención. El análisis de costos en los hospitales es de gran interés, pues permite justificar cambios en la organización y la gestión hospitalaria. Objetivo: Determinar el costo de las lesiones vasculares abdominales por proyectil de arma de fuego para el paciente, su familia y el hospital. Material y métodos: Se llevó a cabo un estudio observacional, descriptivo, prospectivo y longitudinal de pacientes con lesiones vasculares abdominales por proyectil de arma de fuego que ingresaron en el Centro de Trauma de Cruz Roja Mexicana en Ciudad de México entre enero y octubre de 2018. Resultados: Ingresaron 8149 pacientes, 149 con diagnóstico de herida por proyectil de arma de fuego, de los cuales el $0.07 \%$ tuvieron lesión vascular abdominal. El costo total de la atención médica fue en promedio de \$174,770.79 (mediana \$132,999.50) por persona, el cual supera el ingreso anual de esos pacientes e implica una absorción institucional del gasto de hasta el 95.5\%. Conclusiones: La inversión institucional en la atención de patologías con alto impacto y el desarrollo de estrategias para facilitar el acceso a servicios de salud son una necesidad prioritaria real.
\end{abstract}

Palabras clave: Costos. Lesiones vasculares. Trauma penetrante de abdomen.

\begin{abstract}
Background: Violent trauma with penetrating injuries is a relevant public health issue. Penetrating abdominal wounds cause 90-95\% of vascular injuries, which not only have high associated morbidity and lethality, but also involve high attention costs. Cost analysis in hospitals has become a topic of great interest, as it justifies changes in hospital organization and management. Objective: Determine the cost of gunshot abdominal vascular injuries for the patient, his family and the hospital. Material and methods: We drove an observational, descriptive, prospective and longitudinal study of patients with of gunshot abdominal vascular injuries admitted in the Mexican Red Cross Trauma Center in the Federal District from January $1^{\text {st }}$ to October 31'st, 2018. Results: 8149 patients were admitted in the emergency department, 149 with a diagnosis of gunshot injury, of which $6(0.07 \%)$ had abdominal vascular injury. The total cost of medical care these patients was on average $\$ 174,770.79$ (median $\$ 132,999.50$ ) per capita, amount that surpasses their annual income and implies an institutional
\end{abstract}

\section{Correspondecia:}

*Adriana Hernández García

Avda. Ejército Nacional, 1032

Col. Los Morales-Polanco, Del. Miguel Hidalgo Fecha de recepción: 08-12-2019

C.P. 11510, Ciudad de México, México Fecha de aceptación: 30-03-2019

Cir Cir. 2020;88(4):467-472

E-mail: adri.hega@gmail.com

DOI: $10.24875 / C I R U .20001743$

Contents available at PubMed

www.cirugiaycirujanos.com

0009-7411/@ 2019 Academia Mexicana de Cirugía. Publicado por Permanyer. Este es un artículo open access bajo la licencia CC BY-NC-ND (http://creativecommons.org/licenses/by-nc-nd/4.0/). 
absorption of expenditure up to $95.5 \%$. Conclusions: Institutional investment on the attention of high-impact pathologies and the development of strategies that facilitate access to health services are a real and priority necessity.

Key words: Costs. Vascular injuries. Abdominal penetrating trauma.

\section{Introducción}

El trauma violento con lesiones penetrantes (por proyectil de arma de fuego [PAF] o instrumento punzocortante) es un problema de salud pública relevante: se encuentra entre las principales causas de muerte en el mundo y afecta principalmente a la población económicamente activa. En todo el mundo y en hospitales no militares, las lesiones vasculares representan el $0.65 \%$ del total de ingresos anuales en los servicios de urgencias, y en México comprenden el $9 \%$ de esa cifra. Las heridas penetrantes abdominales causan el $90-95 \%$ de las lesiones vasculares abdominales (las cuales se encuentran en el $22-25 \%$ de los pacientes sometidos a laparotomía por heridas abdominales por PAF) y se estima que se asocian con una media de dos a cuatro lesiones adicionales; además, es común observar compromiso de múltiples vasos. En términos generales, la letalidad de las lesiones arteriales es del $35-85 \%$ y la de las lesiones venosas es del $50-90 \%{ }^{1-4}$.

La investigación médica ha demostrado que muchos países de ingresos altos han reducido de manera significativa las tasas de mortalidad del trauma al implementar sistemas sustentados en la mejora de la calidad de la organización y la planificación de la atención desde el ámbito prehospitalario, la reanimación inicial en el hospital y la atención definitiva a corto, mediano y largo plazo. De acuerdo con esta información, evaluar a los pacientes en conformidad con el protocolo ATLS $^{\circledR}$ y prestar pronta atención y adecuado tratamiento quirúrgico, incluyendo la toma de decisión oportuna con respecto a llevar a cabo control de daños, no solo asegura una mayor sobrevida sino que también proporciona un mejor pronóstico global ${ }^{5-10}$.

El análisis de costos en los hospitales es un tema que ha suscitado gran interés en los últimos años, pues es la única manera de justificar esos cambios en la organización y la gestión hospitalarias desde el punto de vista administrativo (cuyo objetivo prioritario es la búsqueda de la eficiencia); dicho de otra manera, los estudios de costos se han vuelto indispensables en el área de la salud (al igual que en cualquier industria) para planificar estos sistemas y su financiamiento. Por otra parte, la situación epidemiológica y la escasez de recursos de los países de bajos y medianos ingresos implican evaluar diferentes medios para implementar estos cambios con el menor costo posible. Por estos motivos, cada vez es más necesario fundamentar la toma de decisiones en estudios con perspectiva médica y económica ${ }^{11-18}$.

Consideramos relevante efectuar un estudio con esta perspectiva sobre las lesiones vasculares abdominales por PAF por dos motivos: 1) este tipo de trauma se considera de alto impacto porque implica unas altas morbilidad y letalidad debidas al estado de choque y las lesiones asociadas, y 2) la identificación y la contención del costo de producción de los servicios de salud han sido poco estudiadas en México y son aspectos cruciales para organizar y optimizar recursos con el fin de obtener buenos resultados.

\section{Método}

Se llevó a cabo un estudio observacional, descriptivo, prospectivo y longitudinal de los pacientes con lesiones vasculares abdominales por PAF que ingresaron en el Centro de Trauma de Cruz Roja Mexicana en Ciudad de México del 1 de enero al 31 de octubre de 2018, con el fin de determinar el costo de este tipo de trauma para el paciente, su familia y el hospital. Captamos a todos los pacientes con diagnóstico de ingreso por herida de PAF en el abdomen e identificamos a aquellos con diagnóstico posquirúrgico de lesiones vasculares abdominales; posteriormente construimos un registro a partir de fuentes secundarias (expediente clínico físico y expediente electrónico radiológico y de estudios de laboratorio), donde capturamos los recursos que generaron gasto a los pacientes y, con ayuda del área administrativa del hospital, determinamos los costos de la atención médica hospitalaria. Finalmente, revisamos las notas de trabajo social para conocer el nivel socioeconómico y los ingresos de los pacientes, y así tener un punto de comparación para determinar el impacto económico de la lesión en el paciente y su familia.

\section{Resultados}

Durante el periodo de estudio ingresaron 8149 pacientes en el área de urgencias del Centro de Trauma de 
Cruz Roja Mexicana en Ciudad de México, 149 (1.82\%) con diagnóstico de ingreso de herida por PAF, de los cuales $21(0.25 \%)$ tuvieron diagnóstico posquirúrgico de lesión visceral aislada o asociada con otros sistemas y $6(0.07 \%)$ de lesión vascular abdominal. Ocurrieron $94(1.15 \%)$ defunciones, $15(0.18 \%)$ correspondientes a pacientes con herida por PAF en el abdomen y $3(0.03 \%)$ con lesión vascular abdominal. El tiempo de traslado de los pacientes osciló entre 25 y 60 minutos (media de 37.5 ) y el $100 \%$ de los pacientes con el diagnóstico de interés ingresaron en cubículo de choque.

Todos los pacientes con lesión vascular abdominal por PAF fueron de sexo masculino, con una edad de 23 a 52 años (media de 37.5). En lo referente a las lesiones intraabdominales asociadas, un paciente (16.6\%) tuvo lesión en dos órganos, tres (50\%) en cuatro órganos, uno (16.6\%) en cinco órganos y uno (16.6\%) en seis órganos (Tabla 1). El $83.3 \%$ de los pacientes tuvieron lesión en un solo vaso y el $16.6 \%$ en dos vasos; el $50 \%$ tuvieron lesión en la vena cava inferior, el $33.3 \%$ en la arteria iliaca, el $16.6 \%$ en la aorta y el $16.6 \%$ en la vena iliaca. El tratamiento de la lesión vascular consistió en rafia $(66.6 \%)$ o ligadura (33.3\%). El sangrado durante el primer evento quirúrgico fue de 2000-5000 ml, con un promedio de $4080 \mathrm{ml}$.

El tiempo quirúrgico de las intervenciones iniciales osciló entre 50 y 240 minutos (promedio de 159.8). El $50 \%$ de los pacientes solo ameritaron un evento quirúrgico y el $50 \%$ debieron ser sometidos a desempaquetamiento, laparorrafia, second look $\mathrm{o}$ aseo quirúrgico: uno (16.6\%) requirió seis intervenciones quirúrgicas, otro (16.6\%) tres, y uno más (16.6\%) dos. El $50 \%$ de los pacientes ingresaron en la unidad de cuidados intensivos, donde permanecieron durante 5-14 días (promedio de 8.6; mediana de 6). El tiempo de estancia hospitalaria total osciló entre 1 y 45 días (promedio de 12.5; mediana de 9).

El análisis de costos de la enfermedad se efectuó mediante la cuantificación de los recursos médicos utilizados generadores de gastos y la suma de sus precios (Tabla 2). El costo total de la atención médica de los pacientes con lesión vascular abdominal por PAF fue de $\$ 1,048,624.79$ (promedio de $\$ 174,770.79$; mediana de \$132,999.50) por paciente (mínimo de $\$ 27,727.64$; máximo de $\$ 493,103.47$ ) (Tabla 3).

Los estudios socioeconómicos demostraron que el $50 \%$ de los pacientes tenía familia conformada por tres personas, el $33.3 \%$ por dos y el $16.6 \%$ por integrante único. La escolaridad del $66.6 \%$ de los pacientes fue secundaria completa, del $16.6 \%$ preparatoria incompleta y del $16.6 \%$ carrera técnica. La fuente de
Tabla 1. Lesiones de órganos abdominales por herida por proyectil de arma de fuego asociadas con lesiones vasculares

\begin{tabular}{lc}
\hline Órgano lesionado & Porcentaje \\
\hline Intestino delgado & $83.3 \%$ \\
Colon & $50 \%$ \\
Hígado & $33.3 \%$ \\
Uréter & $16.6 \%$ \\
Vía biliar & $16.6 \%$ \\
Riñón & $16.6 \%$ \\
Estomago & $16.6 \%$ \\
Bazo & $16.6 \%$ \\
Fractura de cuerpo vertebral & $16.6 \%$ \\
Fractura de sacro & $16.6 \%$ \\
\hline
\end{tabular}

Tabla 2. Cantidad de recursos utilizados en la atención médica en seis pacientes con diagnóstico de lesión vascular abdominal por proyectil de arma de fuego

\begin{tabular}{lccc}
\hline Recurso que generó costo & Mínimo & Máximo & Promedio \\
\hline $\begin{array}{l}\text { Pacientes ingresados a área de } \\
\text { choque }\end{array}$ & 6 & 6 & 6 \\
$\begin{array}{l}\text { Días de estancia en piso } \\
\begin{array}{l}\text { Días de estancia en unidad de } \\
\text { cuidados intensivos }\end{array}\end{array}$ & 0 & 45 & 7.8 \\
$\begin{array}{l}\text { Número de exámenes de } \\
\text { laboratorio }\end{array}$ & 5 & 214 & 4.3 \\
$\begin{array}{l}\text { Número de estudios de gabinete } \\
\text { Número de procedimientos }\end{array}$ & 0 & 26 & 11.8 \\
quirúrgicos & 1 & 6 & 2.3 \\
\hline
\end{tabular}

ingresos del $83.3 \%$ de los pacientes provenía del ejercicio de algún oficio (comerciante, albañil, plomero) y la del $16.6 \%$ de una carrera técnica. El $66.6 \%$ de los pacientes se clasificaron como nivel socioeconómico 1 y el $33.3 \%$ como nivel socioeconómico 0 (el $83.3 \%$ de los pacientes tenía ingresos menores que un salario mínimo y el $16.6 \%$ mayores).

\section{Discusión}

\section{Relevancia médica del trauma vascular abdominal}

Como se mencionó en la introducción, las lesiones vasculares representan el $0.65 \%$ del total de los 
Cirugía y Cirujanos. 2020;88(4)

Tabla 3. Costos de la atención médica en seis pacientes con diagnóstico de lesión vascular abdominal por proyectil de arma de fuego

\begin{tabular}{lccc}
\hline Recurso que generó costos & Costo mínimo (\$) & Costo máximo (\$) & Costo promedio (\$) \\
\hline Costos por los días de estancia en urgencias & 1,200 & 3,703 & $2,458.86$ \\
Costos por los días de estancia en piso & $15,209.18$ & $167,428.27$ & $49,745.44$ \\
Costos por los días de estancia en unidad de cuidados intensivos & 17,500 & 42,000 & 14,000 \\
Costos por exámenes de laboratorio & 537.93 & 110,700 & $37,303.96$ \\
Costos por exámenes de gabinete & 500 & 5,400 & $2,133.33$ \\
Costos por procedimiento quirúrgico & 19,660 & $61,512.50$ & $45,022.5$ \\
Costo total de atención médica & $27,727.64$ & $493,103.47$ & $174,770.79$ \\
Costo cubierto por el paciente & 200 & 20,000 & $10,905.66$ \\
\hline
\end{tabular}

ingresos anuales en los servicios de urgencias en el mundo, y en México comprenden el $9 \%^{2}$. En este estudio, el $0.07 \%$ de los ingresos en urgencias fueron por lesión vascular abdominal por PAF. Esta cantidad es menor que la reportada por Rodríguez-López et al., ${ }^{2}$ pero no todos los pacientes incluidos en ese estudio tenían lesiones en el abdomen de manera exclusiva, lo cual podría explicar la discrepancia entre las cifras. Heuer, et al. ${ }^{18}$ reportaron 10,530 (el 1.5\% de los ingresos en urgencias) casos de pacientes con trauma abdominal con lesiones vasculares graves en 218 hospitales, durante 16 años, en Alemania; en este caso, la diferencia podría deberse al número de hospitales incluidos y el tiempo dedicado al estudio. En nuestro país, una referencia comparativa, de un centro de trauma, es el propio Centro de Trauma de Cruz Roja Mexicana en Ciudad de México: en un trabajo retrospectivo entre 2015 y $2017^{19}$ se reportaron 42,909 ingresos en el área de urgencias, el 1.4\% con diagnóstico de herida por PAF, del cual el $0.19 \%$ tuvo heridas en el abdomen y el $0.02 \%$ lesión vascular abdominal; todas estas cifras son menores que las encontradas en 2018. Estos datos parecen indicar que la incidencia de este tipo de trauma se ha incrementado de manera progresiva a lo largo de los últimos años.

Asensio, et al. ${ }^{3}$ estimaron que las lesiones vasculares abdominales se asocian con una media de dos a cuatro lesiones adicionales; en nuestro estudio observamos que el $100 \%$ de los pacientes tuvieron lesiones abdominales asociadas, con una media de 4.1; cantidad similar al límite alto reportado en el artículo referido. Asensio, et al. ${ }^{3}$ también reportaron que la lesión vascular múltiple es frecuente; sin embargo, nosotros encontramos que el $83.3 \%$ de los pacientes tuvieron lesión en un solo vaso. Es evidente que la relevancia de las lesiones adicionales no solo yace en el incremento global del índice de gravedad de la lesión, sino también en la complejidad y la duración del tratamiento requerido, así como en el riesgo de desarrollar complicaciones ${ }^{6,8,20,21}$.

De acuerdo con Heuer, et al. ${ }^{18}$ y Karaolanis, et al..$^{20}$, más del $70 \%$ de las muertes de estos pacientes ocurrirán durante las primeras 24 horas. En nuestro estudio, el $66.6 \%$ de los fallecimientos sucedió en el primer día y el $33.3 \%$ después de 9 días por complicaciones como falla orgánica múltiple y sepsis, de manera similar a lo establecido en los artículos referidos. Rodríguez, et al. ${ }^{4}$ estimaron la letalidad de las lesiones arteriales en un 35-85\% y de las venosas en un $50-90 \%$, y Asensio, et al..$^{21}$ reportaron una letalidad del $79 \%$ para lesiones aisladas de aorta y del $75 \%$ para lesiones de vena cava inferior; nuestro estudio encontró una letalidad del 100\% para lesiones de aorta y del $50 \%$ para lesiones de vena cava inferior, cifras elevadas que no difieren de manera considerable de las reportadas en la literatura consultada. En cuanto a la referencia comparativa nacional, en el Centro de Trauma de Cruz Roja Mexicana en Ciudad de México observamos un incremento en la letalidad de las lesiones vasculares abdominales por PAF: del $0.01 \%$ en $2015-2017$ al $0.03 \%$ en 2018.

\section{Relevancia económica del trauma vascular abdominal}

Heuer, et al. ${ }^{18}$ reportaron un $74.2 \%$ de lesiones vasculares abdominales por PAF en el sexo masculino, 
con una edad de $41.7 \pm 19.2$ años; en nuestro estudio encontramos el $100 \%$ en el sexo masculino, con una edad de $37.5 \pm 14.3$ años. De acuerdo con el Instituto Nacional de Estadística y Geografía (INEGI), la edad media en nuestro país en 2015 era de 27 años; si consideramos que esta cifra no ha cambiado de manera considerable a lo largo de los últimos 3 años, es fácil ver por qué está contenida en el rango de edad de los pacientes con lesiones vasculares abdominales por $\mathrm{PAF}^{22}$. En cuanto al nivel de instrucción, el área de educación del INEGI reportó que el $33.5 \%$ de la población tenía un nivel medio-superior o superior, el $37.9 \%$ secundaria completa, el $19.5 \%$ primaria completa y el $8.9 \%$ primaria incompleta ${ }^{22}$; nuestro estudio encontró que el $66.6 \%$ de los pacientes con lesiones vasculares abdominales por PAF tenían secundaria completa, el $16.6 \%$ preparatoria incompleta y el $16.6 \%$ carrera técnica, resultados que también reflejan la situación de la población de nuestro país.

La Encuesta Nacional de Ocupación y Empleo de 2010-2011 reportó que el nivel de ingresos en las áreas más urbanizadas es de un salario mínimo ( $\$ 88.36$ diarios) en el $13.4 \%$ de la población, de más de uno hasta dos salarios mínimos en el $61.5 \%$ y que el $8.4 \%$ no percibe ingresos ${ }^{22}$; nuestro estudio reveló que el $83.3 \%$ de los pacientes reportaron un ingreso menor de un salario mínimo y el $16.6 \%$ mayor de un salario mínimo, cifras que demuestran que la población que recibe los servicios del Centro de Trauma de Cruz Roja Mexicana en Ciudad de México tiene ingresos muy por debajo de la media nacional.

De acuerdo con la Organización para la Cooperación y el Desarrollo Económico (OCDE), hasta 2016 casi el $60 \%$ de los empleos en el país pertenecían al sector informal y cerca del $22 \%$ de los jóvenes no contaban con empleo formal, estudiaba o recibía algún tipo de capacitación, comparado con el 15\% en los países de la $\mathrm{OCDE}^{23}$. En nuestro estudio encontramos que la fuente de ingresos del $83.3 \%$ de los pacientes provenía de algún oficio (comerciante, albañil, plomero, etc.) y del $16.6 \%$ de una carrera técnica. Resulta evidente que las altas tasas de empleo informal inevitablemente limitan los ingresos y, en consecuencia, los recursos disponibles para financiar la atención a la salud. Por otra parte, el INEGI reportó que solo el $34.8 \%$ de la población contaba con acceso a instituciones públicas de salud ${ }^{22}$; en nuestro estudio, ningún paciente contaban con servicio médico institucional, lo cual, sumado a los argumentos antes presentados, también impacta de manera desfavorable sobre la disponibilidad de recursos terapéuticos.
En 2004, Cowey, et al. ${ }^{24}$ llevaron a cabo una revisión de 187 pacientes con herida por PAF ingresados en un hospital escuela en Inglaterra en un periodo de 54 meses y encontraron que el costo total de la atención ascendía a £272,000 (equivalente a $\$ 6,800,000$ ), con un promedio de $£ 1,428$ (equivalente a $\$ 35,700)^{24}$, cifra mayor que la obtenida en nuestro estudio, donde el costo total de la atención encontrado fue de $\$ 1,048,624.79$, aunque con un promedio de $\$ 174,770.79$. La discrepancia entre las cifras podría deberse a que nuestro tiempo de estudio fue de 10 meses y que solo contemplamos pacientes con lesiones vasculares abdominales por PAF; no obstante, es claro que las cantidades exceden el ingreso anual promedio de la población que recibe los servicios del Centro de Trauma de Cruz Roja Mexicana en Ciudad de México (aproximadamente \$32,251.40).

En nuestro Centro de Trauma de Cruz Roja Mexicana, que es una institución de asistencia privada sin fines de lucro, ningún paciente cubre el costo total de la atención. De acuerdo con nuestro estudio, el paciente con menor aportación cubrió el $0.5 \%$ del costo total de la atención y el paciente con mayor aportación cubrió el 25\%. Estos resultados demuestran que cada paciente con lesiones vasculares abdominales por PAF, que en conjunto representan el $0.07 \%$ de los ingresos en urgencias durante 10 meses y, en consecuencia, el $0.002 \%$ del gasto institucional total en el mismo periodo, implica un gasto institucional promedio de $\$ 148,555.17$. Si extrapolamos esta información a las instituciones públicas de salud, que cubren prácticamente el $100 \%$ del costo total de la atención, o a los hospitales privados, donde el paciente cubre el $100 \%$ del costo total de la atención, podemos observar la magnitud del gasto que implica este tipo de lesiones, sin tomar en consideración la variabilidad en los precios de venta al público de los recursos materiales necesarios para su atención o en las percepciones del personal encargado de la misma.

\section{Conclusiones}

Si bien las lesiones vasculares abdominales por PAF son poco frecuentes, constituyen un problema de salud pública relevante por los siguientes motivos: 1) elevada mortalidad; 2) alto riesgo de desarrollar complicaciones graves que ameritan gran cantidad de estudios de laboratorio y gabinete, tratamientos complejos y especializados, y estancias hospitalarias prolongadas en áreas de medicina 
crítica; 3) afecta principalmente a la población económicamente activa; y 4) el costo total de la atención excede el ingreso anual promedio de esa población, y aunque solo representa una pequeña parte del gasto institucional total, definitivamente nunca será comparable con el valor de la vida de un ser humano. Esta información no solo permite que la comunidad médica conozca la realidad socioeconómica en la cual se desenvuelven sus pacientes con el fin de llevar a cabo valoraciones verdaderamente integrales sobre las posibilidades de recuperación que el ambiente y la familia ofrecen y contribuir a la promoción de una medicina social, integral y más humana, sino que también constituye un argumento para demostrar a la comunidad administrativa que la inversión en la atención de patologías con alto impacto y el desarrollo de estrategias que faciliten el acceso a los servicios de salud son una necesidad real, con resultados que tienen consecuencias en toda la población.

\section{Agradecimientos}

Los autores agradecen a maestros, pacientes y personal que labora en el Centro de Trauma de Cruz Roja Mexicana en Ciudad de México.

\section{Conflicto de intereses}

Los autores declaran no tener ningún conflicto de intereses al realizar este estudio.

\section{Responsabilidades éticas}

Protección de personas y animales. Los autores declaran que para esta investigación no se han realizado experimentos en seres humanos ni en animales.

Confidencialidad de los datos. Los autores declaran que en este artículo no aparecen datos de pacientes.

Derecho a la privacidad y consentimiento informado. Los autores declaran que en este artículo no aparecen datos de pacientes.

\section{Bibliografía}

1. Rodríguez C, Vázquez R. El inicio de la laparotomía en el trauma abdominal en México. Cirujano General. 2001;23:278-81.

2. Rodríguez-López E, Fabián-Mijangos W, Casares-Bran T, Lecuona-Huet N, Olivares-Cruz S, Carbajal-Robles V, et al. Trauma vascular civil: tres años de manejo en el Hospital General de México. Revista Mexicana de Angiología. 2017;45:154-2.

3. Asensio J, Petrone P, Karsidag T, Demiray S, Kuncir E. Lesiones vasculares abdominales. Un desafío mayor para el cirujano de trauma. Cirujano General. 2003;25:238-46.

4. Rodríguez F, Vivas L, Ottolino P, Viteri Y, Basilio A. Lesiones vasculares abdominales. Algunos consejos transoperatorios. Trauma. 2006;9:42-7.

5. Camacho J, Mascareño S. Cirugía de control de daños. Gac Med Mex. 2013;149:61-72

6. Leonher K, Jiménez J, Ramírez L, Santa M, Gil J, Tello I. Trauma abdominal cerrado y penetrante con lesión a órganos abdominales. Revista Latinoamericana de Cirugía. 2013;3:20-3.

7. Vega J, Velázquez J, Vega-Pérez J, Yáñez J, Zaldivar G, Guerrero G. Cirugía de control de daños. Cirujano General. 2010;32:37-42.

8. Basilio A, Olguín A, Delgadillo S, López O. Reintervención en trauma abdominal. Trauma. 2005;8:5-9.

9. Sánchez J, Menéndez P, Asensio J. Lesiones vasculares abdominales. Cirugía Española. 2012:90:215-21.

10. Asensio J, Navarro S, Forno W, Roldán G, Petrone P, Gambaro E, et al. Abdominal vascular injuries: the trauma surgeon's challenge. Surgery Today. 2001;31:949-57.

11. Santamaría BM, Herrera JE, Sil PA, Santamaría NH, Flores MA, Del Arco A. Estructura, sistemas y análisis de costos de la atención médica hospitalaria. Medicina e Investigación. 2015;3:134-40.

12. Irena $T$, Higuera F. Costo beneficio: algunas experiencias en el Hospital General de México. Rev Mex Cardiol. 2001;12(12):94-9.

13. Jiménez J, Cid $C$. Conocer costos en atención a la salud: una necesidad imperativa. Rev Chil Cardiol. 2011;30:227-9.

14. Arrendo A, Damián T. Costos económicos en la producción de servicios de salud: del costo de los insumos al costo del manejo de casos. Salud Pública de México. 1997;39:117-24.

15. Rodríguez E. Revistas de ciencias administrativas y financieras de seguridad social. Rev Cienc Financ Segur Soc. 2000;8:1-5

16. Loredo-Abdalá A, Casas-Muñoz A, González-Garay A, Ortiz-Hernández R, González-Corona J, Ramírez-Angoa L. Trauma craneal no accidental en niños: costo económico directo de la atención médica en un hospital de tercer nivel. Gac Med Mex. 2018;154:671-80.

17. Silva M, Brain M. Validez y confiabilidad del estado socioeconómico. Salud Pública y Trabajo Social. 2015;1-350.

18. Heuer M, Hussmann B, Kaiser M, Lefering R, Paul A, Lendemans S. Abdominal vascular trauma in 760 severely injured patients. Eur $\mathrm{J}$ Trauma Emerg. 2013;39:47-55.

19. Trueba Lozano D. Propuesta para evaluar la calidad en la atención del paciente traumatizado en el Centro de Trauma de Cruz Roja Mexicana en el Distrito Federal. Universidad Nacional Autónoma de México. Ciudad de México, México, 2019.

20. Karaolanis G, Moris D, McCoy C, Tsilimigras D, Georgopoulos S, Bakoyiannis $\mathrm{C}$. Contemporary strategies in the management of civilian abdominal vascular trauma. Frontiers in Surgery. 2018:5:1-11.

21. Asensio J, Chahwan S, Hanpeter D, Demetriades D, Forno W, Gambaro $\mathrm{E}$, et al. Operative managment and outcome of 302 abdominal vascular injuries. Am J Surg. 2000;180:528-34.

22. Encuesta Nacional de Ocupación y Empleo. ENOE 2010 Instituto Nacional de Estadística y Geografía, Secretaría del Trabajo y Previsión Social. México: INEGI; 2011.

23. Estudios de la OCDE sobre los Sistemas de Salud: México 2016. Organización para la Cooperación y el Desarrollo Económicos. Ciudad de México, México, 2016.

24. Cowey A, Mitchell P, Maclennan I, Pearson R. A review of 187 gunshot wound admissions to a teaching hospital over a 54-month period: training and service implications. Ann R Coll Surg Engl. 2004;86:104-7. 